\title{
Medición y análisis de la impedancia en PLC de banda angosta en un ambiente universitario
}

\author{
Measurement and analysis of Narrowband PLC impedance in a University environment \\ Matías N. Hadad ${ }^{\dagger 1}$, Patricio G. Donato ${ }^{\dagger 2}$ y Marcos A. Funes ${ }^{\dagger 3}$ \\ ${ }^{\dagger}$ Instituto de Investigaciones Científicas y Tecnológicas en Electrónica (ICYTE) \\ Consejo Nacional de Investigaciones Científicas y Técnicas (CONICET) \\ Universidad Nacional de Mar del Plata (UNMDP) \\ Juan B. Justo 4302, Mar del Plata, Argentina \\ 1 mhadadefi.mdp.edu.ar \\ ${ }^{2}$ donatopgefi.mdp.edu. ar \\ 3 mfunesefi.mdp.edu.ar \\ Recibido: 03/03/21; Aceptado: 28/03/21
}

\begin{abstract}
Resumen-Los sistemas de comunicación por la red eléctrica de banda angosta son ampliamente utilizados en aplicaciones como medidores inteligentes, automatización de hogares, y sistemas de control y comando de baja velocidad, entre otras. Sin embargo, las redes eléctricas no fueron diseñadas para transmitir datos y representan un ambiente hostil para las comunicaciones. En la bibliografía específica se encuentran estudios que evidencian las diversas problemáticas de las comunicaciones por la red eléctrica de banda angosta, siendo la impedancia una de las más importantes. El principal objetivo de este trabajo es proveer un estudio de la impedancia de la red eléctrica para esta banda de comunicación. Este estudio fue realizado en un ambiente Universitario que contiene un número diverso de cargas desconocidas, considerando las variaciones con respecto al tiempo y la ubicación. Se describe el sistema de adquisición desarrollado a medida para hacer el relevamiento. El análisis de los resultados muestra que la impedancia de la red presenta problemas para la comunicación PLC al menos tan severos como los relevados en otros estudios realizados en diferentes países y entornos.
\end{abstract}

Palabras clave: PLC de banda angosta; Impedancia; Campaña de Medición.

Abstract-Narrowband powerline communication systems are widely used in many applications, such as smart metering devices, home automation and low-speed command and control systems. However, power lines were not designed for data transmission and they represent a harsh environment for communication. In the specific bibliography, it is possible to find studies that show the diverse issues of Narrowband Powerline Communication, being the impedance of the grid one of them. The main objective of the present work is to provide a study on the impedance of the grid over this communication band. This study was done in a University environment which has a diverse number of unknown loads, considering the variation with time and location. The data acquisition system developed to conduct the study is shown. The analysis of the results shows that the local grid exhibits issues as least as severe as the ones presented by other authors in different countries and environments.

Keywords: Narrowband PLC; Impedance; Measurement Campaign

\section{INTRODUCCIÓN}

Durante la última década se ha producido una evolución en las redes eléctricas del mundo, principalmente impulsada por el concepto de las redes eléctricas inteligentes, que involucra desafíos tecnológicos importantes. Uno de los conceptos principales de la redes eléctricas inteligentes es el empleo de sistemas de comunicaciones que interconecten a todos los dispositivos de la red. Estos sistemas deben soportar tanto la comunicación entre consumidores y proveedores como la necesidades de los sistemas de automatización y monitoreo de la red eléctrica. Por este motivo, las investigaciones en sistemas de comunicación han recibido un fuerte impulso. Entre las diversas opciones disponibles, la tecnología de las comunicaciones por la red eléctrica (PLC, del inglés Power Line Communications) ha despertado un gran interés por parte de la industria y las empresas de servicios [1]. Uno de los atractivos de esta tecnología respecto de otras técnicas de comunicación es la existencia de un soporte físico, la red eléctrica, que sirve no solo para distribuir energía sino también para transmitir datos. Este interés ha sido apoyado por entes regulatorios mediante la publicación de estándares como IEEE 1901.2 [2] en 2013 y IEEE 1901.2a [3] en 2015, para frecuencias menores a $500 \mathrm{kHz}$.

Desde un punto de vista académico e industrial, PLC es dividido en dos bandas principales, con diferentes aplicaciones específicas, determinadas por el rango de frecuencia utilizado. Las comunicaciones que usan frecuencias menores a $500 \mathrm{kHz}$ son categorizadas como PLC de banda angosta (NB-PLC, del inglés Narrowband $P L C)$ y son principalmente utilizadas para enviar y recibir señales de control y telemetría entre dispositivos y centrales de control, o para la transmisión de datos de información de baja velocidad, como ser datos de medidores inteligentes que usualmente reportan en intervalos de 15 o 30 minutos. La segunda banda es conocida como PLC de banda ancha (BB-PLC del inglés Broadband PLC) que comprende todas las comunicaciones en frecuencias mayores a $500 \mathrm{kHz}$ y son principalmente utilizadas para transmitir y recibir video o para redes de computadoras. Pese a que el medio físico 
es el mismo, las problemáticas que presentan cada una de estas bandas es diferente.

En el campo de las redes eléctricas inteligentes, para las comunicaciones con medidores inteligentes o inversores conectados a la red, NB-PLC es la banda más interesante debido a que requiere solamente un volumen moderado de datos. Las principales problemáticas que se encuentran en esta banda son la presencia de ruido no Gaussiano con comportamiento impulsivo [4] y la impedancia de la red eléctrica. Los efectos del ruido han sido analizados en profundidad en diversos trabajos de la literatura científica [5], mientras que los problemas producidos por la impedancia no han sido sido estudiados con la misma profundidad. Dado que la impedancia es el resultado de la topología de la red y del tipo y cantidad de cargas conectadas, su valor depende de la instalación particular, el tiempo y la posición, y puede ser menor a los valores considerados por equipos comerciales y notas de aplicación de fabricantes de circuitos integrados. Por esta razón, para diseñar correctamente el circuito de acoplamiento de un módem PLC, el valor de la impedancia debe ser correctamente evaluado [6], [7].

Los sistemas NB-PLC son clasificados en sub-bandas dependiendo de la normativa considerada. Para Europa, se definen las las siguientes bandas (EN 50065-1 [8]):

- CENELEC A: $9 \mathrm{kHz}$ a $95 \mathrm{kHz}$

- CENELEC B: $95 \mathrm{kHz}$ a $125 \mathrm{kHz}$

- CENELEC C: $125 \mathrm{kHz}$ a $140 \mathrm{kHz}$

- CENELEC D: $140 \mathrm{kHz}$ a $148 \mathrm{kHz}$

Mientras tanto, IEEE 1901.2 [2], que combina diferentes estándares, define estas otras bandas:

- CENELEC A: $35.9375 \mathrm{kHz}$ a $90.625 \mathrm{kHz}$

- FCC-Low and ARIB 1: $37.5 \mathrm{kHz}$ a $117.1875 \mathrm{kHz}$

- CENELEC B: $98.4375 \mathrm{kHz}$ a $121.875 \mathrm{kHz}$

- ARIB 2 : $154.6875 \mathrm{kHz}$ a $403.125 \mathrm{kHz}$

- FCC-above-CENELEC: $154.6875 \mathrm{kHz}$ a $487.5 \mathrm{kHz}$

Este trabajo trata la problemática y análisis de la medición de impedancia en entornos reales. El objetivo es comprender el comportamiento de la impedancia en el ámbito de una universidad/laboratorio de investigación, que puede ser considerado un ambiente semi-industrial, y evidenciar la limitación que puede presentar el equipamiento comercial en estas condiciones. Se realizó una campaña de medición en el Laboratorio de Instrumentación y Control del Instituto de Investigaciones Científicas y Tecnológicas en Electrónica (ICYTE) [9], analizando el rango de frecuencia entre $20 \mathrm{kHz}$ y $440 \mathrm{kHz}$, que cubre casi la totalidad de las frecuencias consideradas por los estándares IEEE y CENELEC. La sección II describe los problemas causados por una incorrecta estimación del valor de la impedancia. La sección III realiza una breve descripción el sistema desarrollado para realizar la campaña de medición. La sección IV muestra y analiza los resultados obtenidos. Finalmente, la sección V contiene las conclusiones del trabajo.

\section{IMPORTANCIA DE UNA CORRECTA ESTIMACIÓN DE LA IMPEDANCIA}

Para poder entender la importancia de la medición de la impedancia, y los potenciales problemas producidos por una estimación incorrecta, es necesario entender primero como es la arquitectura del circuito de acoplamiento. La Fig. 1 muestra un acoplador capacitivo típico, que es el tipo más utilizado para redes eléctricas de baja tensión. Las funciones principales del acoplador son el filtrado de las componentes de alta tensión, proteger los componentes de baja tensión del módem y acoplar las señales de comunicación de alta frecuencia en la red.

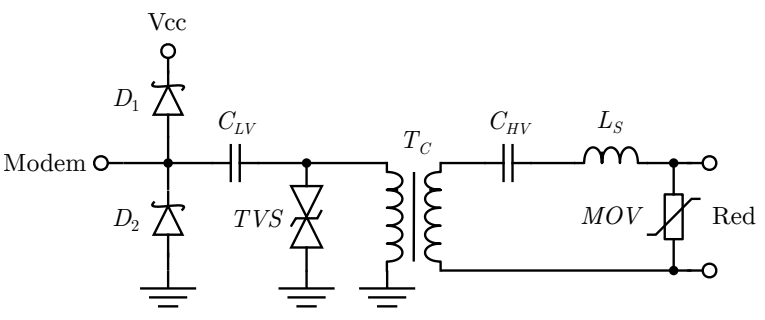

Fig. 1. Acoplador capacitivo típico.

El capacitor de baja tensión $\left(C_{L V}\right)$ permite acoplar la señal de entrada al transformador de acoplamiento $\left(T_{C}\right)$. El capacitor de alta tensión $\left(C_{H V}\right)$ junto con la inductancia de $T_{C}$ actúa como un divisor de tensión hacia la parte de baja tensión del circuito. El inductor $L_{S}$ en serie con $C_{H V}$ permite controlar la frecuencia de resonancia del acoplador, funcionando como un filtro pasabanda. Además de los componentes de acoplamiento antes mencionados, se agregan componentes de protección. Se incluyen diodos de enclavamiento $\left(D_{1}\right.$ y $\left.D_{2}\right)$ para proteger el circuito de baja tensión de sobretensiones, un TVS (Transient Voltage Supressor) es usado para minimizar el transitorio de conexión con la red y un MOV (Metal Oxide Varistor) para limitar las sobretensiones de alta tensión.

La impedancia de la red es muy importante para el diseño de dos partes del circuito, la impedancia serie del filtro de acoplamiento y la etapa de potencia del módem. El caso de una subestimación de la impedancia lleva a un sobredimensionamiento de los componentes del circuito, con la consecuencia de un costo y tamaño mayor al necesario. En el otro extremo, una sobreestimación de la impedancia es más nocivo ya que lleva a problemas de desempeño producidos por la división de impedancia entre la impedancia serie del acoplador y la impedancia de la red. Además, si la etapa de potencia del módem no puede proveer la corriente necesaria, se reduce la potencia efectiva del transmisor.

\section{A. Antecedentes y referencias normativas y comerciales}

Para poder cuantificar la magnitud de la impedancia medida, es necesario conocer las impedancias previstas por normativas y las consideraciones de diseño de dispositivos comerciales y dispositivos presentes en la literatura. A continuación, se presentan varios ejemplos.

El estándar europeo EN 50065-1 [8] define los niveles máximos de tensión para dispositivos que operan en las bandas CENELEC. La sección C.1.2.2 define las características del circuito de prueba para medir estos niveles, el cual es un arreglo en paralelo entre un resistor de $50 \Omega$ y un inductor de $50 \mu \mathrm{H}$.

Janse van Rensburg et al. [10], en su trabajo sobre el diseño de un acoplador integrado, consideran que una red 
eléctrica típica tiene una impedancia de $12.5 \Omega$ y $17.5 \mu \mathrm{H}$ para frecuencias menores a $500 \mathrm{kHz}$.

La nota de aplicación del front-end analógico de bajo costo AFE031 de Texas Instruments [11], muestra el diseño del circuito de acoplamiento para la banda de $23 \mathrm{kHz}$ a $105 \mathrm{kHz}$ especificando un valor $470 \mathrm{nF}$ para $C_{H V}$ y $15 \mu \mathrm{H}$ para $L_{S}$. Teniendo en cuenta estos valores, la impedancia serie del circuito de acoplamiento tiene un rango de $6.67 \Omega$ para el límite superior de la banda y $12.56 \Omega$ para el límite inferior, con una resonancia en $60 \mathrm{kHz}$.

En resultados experimentales realizados localmente con una implementación de la nota de aplicación se observó un comportamiento inadecuado, principalmente producido por tensiones menores a las esperadas en la salida a pesar que la corriente de salida del front-end analógico no llegaba a sus límites. Esto llevó a la hipótesis de que la impedancia de la red eléctrica debía ser menor a la prevista por el diseño.

Diversos trabajos en la literatura muestran resultados que soportan esta hipótesis para diferentes ubicaciones. Cavdar y Karadeniz [12] analizaron la impedancia en ambientes rurales, urbanos e industriales de Turquía, mostrando impedancias en el rango de 5 a $6 \Omega$ para las bandas CENELEC, dependiendo del ambiente. Cortés et al. [13] realizó mediciones de impedancia, en ubicaciones rurales, urbanas y semi-urbanas de España, mostrando valores menores a $5 \Omega$. Bausch et al. [14] analizaron el canal PLC en diferentes emplazamientos domésticos de Alemania, midiendo impedancias tan bajas como $300 \mathrm{~m} \Omega$ y categorizaron el comportamiento en frecuencia de la red eléctrica en 4 tipos característicos.

Teniendo en cuenta los valores obtenidos en estos trabajos, las consideraciones de ninguno de los ejemplos de diseño [8][10][11] concuerda con el comportamiento de la red [12][13][14]. Debido a esto, y para verificar las hipótesis planteada a partir de los ensayos locales con el AFE031, se realizó un estudio de los valores de impedancia en la red local. La siguiente sección muestra una breve descripción del sistema de medición diseñado para este propósito.

\section{SisteMA DE MEDICIÓN}

El sistema diseñado para la medición de impedancia se basa en un circuito de acoplamiento como el de la Fig. 1. Está compuesto por una etapa de potencia, un circuito de acoplamiento, un circuito de medición y un sistema de procesamiento (Fig. 2).

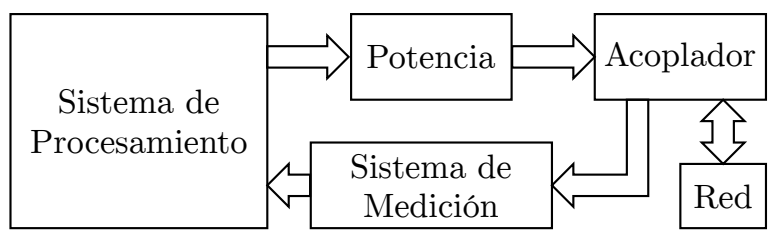

Fig. 2. Diagrama en bloques simplificado del sistema de medición

La operación básica del sistema consiste en la inyección de una señal de prueba, generada por el sistema de procesamiento, a través del circuito de acoplamiento para medir la tensión y corriente, las cuales son usadas por el sistema de procesamiento para calcular la impedancia total. La señal de prueba utilizada es una señal pseudo-aleatoria filtrada y la impedancia es calculada a partir de una FFT de las señales de tensión y corriente capturadas. El valor de impedancia calculado contiene no solo la impedancia de la red sino también la impedancia del circuito de medición, por lo que como parte del procedimiento de medición se realiza una calibración del sistema para estimar correctamente esta impedancia, para tenerla en cuenta en el cálculo de la impedancia de red.

El sistema de procesamiento es implementado con una PC y una placa de desarrollo FPGA Xilinx Spartan 3 Starter Board. La etapa de potencia es implementada con un conversor analógico digital THS5661 de Texas Instruments y un amplificador de potencia OPA561 de Texas Instruments. El sistema de medición consta de circuitos de filtrado diferenciales, un amplificador de ganancia programable y conversores analógico-digitales y son implementados utilizando dos THS4032, dos THS4130 y un THS1206 de Texas Instruments, y un AD8369 de Analog Devices.

La Fig. 3 muestra una foto del prototipo del sistema utilizado para las mediciones, con todos sus bloques funcionales identificados. El sistema permite medir impedancias medias mayores a $300 \mathrm{~m} \Omega$ en el rango de frecuencias de $20 \mathrm{kHz}$ a $440 \mathrm{kHz}$, permitiendo medir mínimos locales tan bajos como $50 \mathrm{~m} \Omega$.

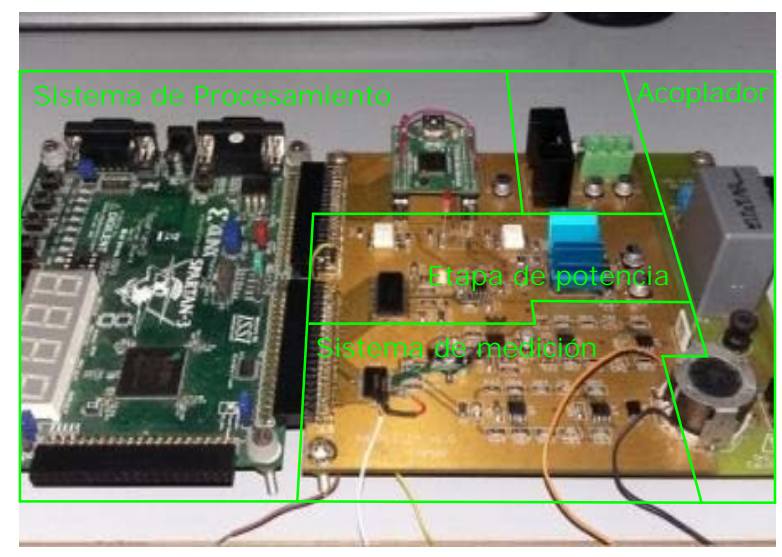

Fig. 3. Foto del prototipo del sistema de medición de impedancia

\section{CAMPaÑa DE MEdición y ANÁlisis de RESULTADOS}

Para verificar la hipótesis planteada sobre los niveles de impedancia, se realizó una campaña de medición en las instalaciones de la Universidad nacional de Mar del Plata. La campaña fue realizada en 3 emplazamientos diferentes del Laboratorio de Instrumentación Control del Instituto de Investigaciones Científicas y Tecnológicas en Electrónica (ICYTE, Argentina), que posee una red de baja tensión trifásica de $220 \mathrm{~V} 50 \mathrm{~Hz}$ con un número desconocido y variable de cargas conectadas. Las mediciones se realizaron en los circuitos internos monofásicos de la institución. El primer emplazamiento, que corresponde a un espacio de trabajo común, fue realizada por 12 días consecutivos, desde el 8/3/2019 al 20/3/2019. La segunda medición fue realizada en el punto común de conexión del laboratorio, durante 8 horas y finalmente, la tercera ubicación, realizada dentro de un tablero que contiene inversores conectados a la red, fue realizada por 3 horas. En cada una de estas mediciones, la 
impedancia fue continuamente analizada y almacenada en intervalos de 5 minutos, guardando los valores, mínimo y medio del intervalo.

La Fig. 4 muestra un gráfico 3D del módulo de la impedancia media medida en la primera ubicación, con el tiempo y la frecuencia como variables independientes. Esta figura muestra que la impedancia de la banda superior no presenta cambios significativos a lo largo de la medición. En la Fig. 4 también se puede ver que el comportamiento predominante respecto a la frecuencia (para frecuencias mayores a $150 \mathrm{kHz}$ ) se corresponde con un sistema inductivo, lo que es esperable.

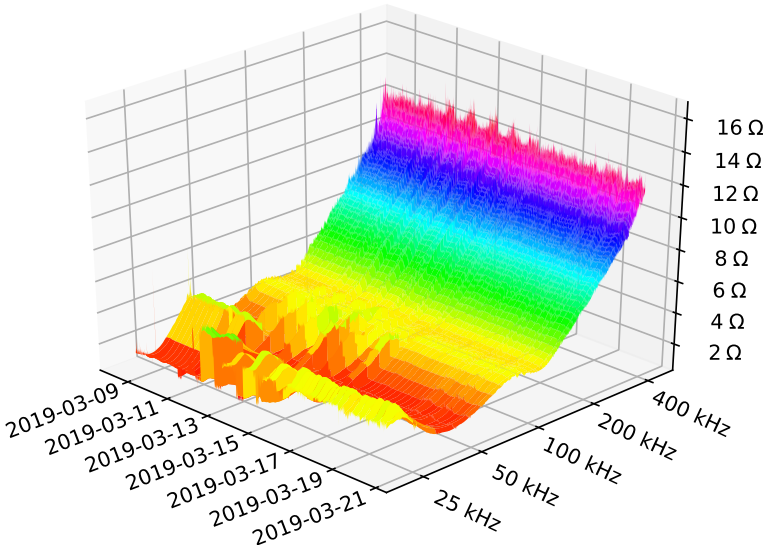

Fig. 4. Comportamiento del módulo de la impedancia media respecto del tiempo y la frecuencia en el primer emplazamiento.

La banda inferior muestra un comportamiento que depende del día, hora y cargas conectadas. Durante el experimento, el jueves 14/3, se cambiaron algunas de las fuentes de alimentación que se hallaban conectadas en las inmediaciones del punto de medición. Este cambio se evidencia más claramente en las mediciones correspondientes a los fines de semana, donde la actividad laboral decrece al mínimo. El primer fin de semana, que corresponde al $9 / 3$ y 10/3, muestra un pico en la impedancia cercano a los $50 \mathrm{kHz}$ y un valle a frecuencias menores. El segundo fin de semana, que corresponde al 16/3 y 17/3 muestra impedancias mayores a baja frecuencia y un valle en $50 \mathrm{kHz}$.

La Fig. 4 también muestra cambios respecto al día y hora. En los períodos de actividad pico, que corresponden al horario entre las 9 y las 17 de lunes a viernes, la impedancia muestra diversos cambios, mientras que para algunas frecuencias la impedancia es mayor, es menor para otras. Este fenómeno, al igual que los anteriores, se puede ver en mas detalle en Fig. 5, que muestra la impedancia media respecto al tiempo para diferentes frecuencias.

En esta figura, los diferentes comportamientos mencionados previamente se pueden ver para las frecuencias de $25 \mathrm{kHz}$ y $102 \mathrm{kHz}$ entre los días $11 / 3$ y $15 / 3$. La impedancia en $25 \mathrm{kHz}$ es menor para las horas de actividad, mientras que la impedancia para $102 \mathrm{kHz}$ es mayor. También se puede ver el cambio de comportamiento a partir del cambio de las fuentes de alimentación empezando la tarde del 14/3. Finalmente, es importante destacar que para frecuencias menores a $64 \mathrm{kHz}$ la impedancia media es menor a $3 \Omega$, que es mucho menor a la impedancia considerada en la red de

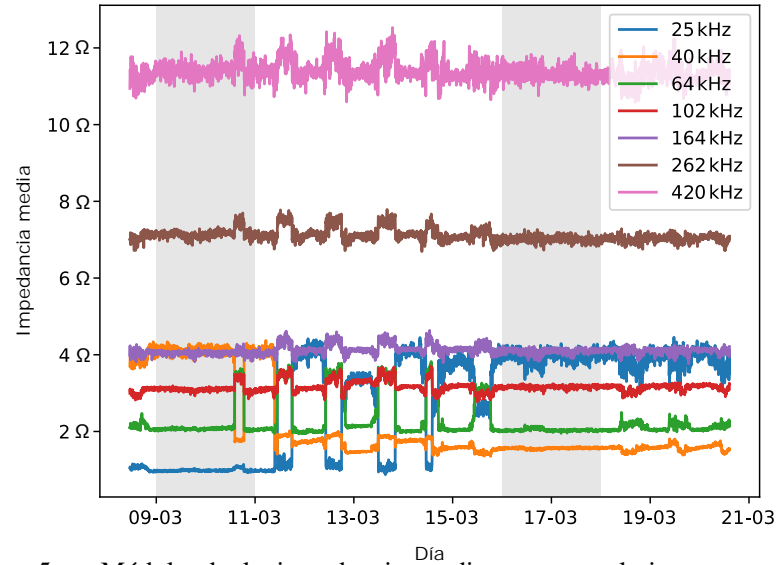

Fig. 5. Módulo de la impedancia media respecto al tiempo para un conjunto de frecuencias en la primera ubicación. En gris se resaltan los fines de semana

prueba de la normativa CENELEC y las notas de aplicación comerciales [8][10][11].

La Fig. 6 muestra la impedancia media respecto de la frecuencia registrada a las $12 \mathrm{pm}$, para diferentes días. En esta figura, al igual que lo mostrado en la Fig. 4, la impedancia no varía significativamente entre días de actividad (días de semana) y de no actividad (fin de semana) para frecuencias mayores a $150 \mathrm{kHz}$, mostrando un comportamiento inductivo.

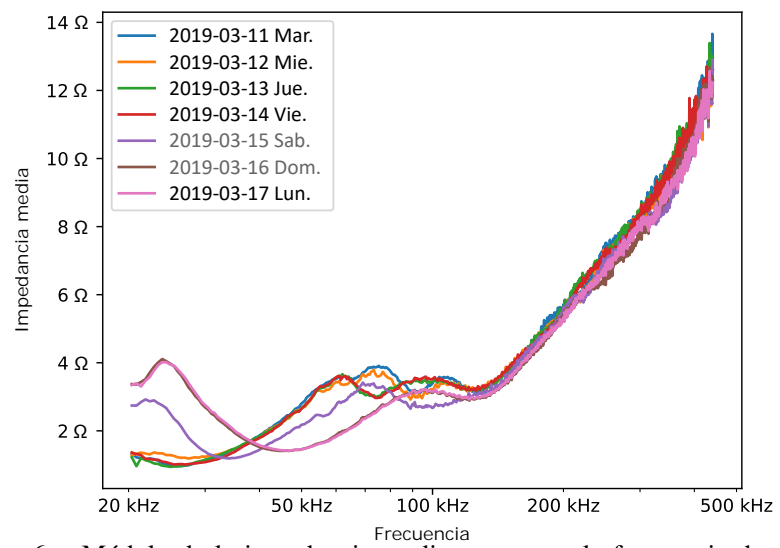

Fig. 6. Módulo de la impedancia media respecto a la frecuencia durante una semana medida a las $12 \mathrm{pm}$ en la primera ubicación

El módulo de la impedancia en la banda de frecuencias más bajas muestra una variación que depende de las cargas conectadas, que se puede ver más claramente en el trazo que corresponde al día 15/3, comparado con los correspondientes a los otros días de la semana. La dependencia con el nivel de actividad se puede notar al comparar los trazos que corresponden al fin de semana del resto de la semana. Esto también se verifica si se analiza la impedancia media respecto a la frecuencia para diferentes horas de un día de la semana (Fig. 7). El comportamiento observado en este emplazamiento no se corresponde directamente con ninguno de los presentados por Bausch et al. [14] ya que el número de mínimos y máximos locales cambia respecto del tiempo. Esto se puede deber a que las mediciones de Bausch et al. [14] fueron realizadas en ambientes residenciales mientras que las de este trabajo corresponden a un ambiente semi-industrial, el cual posee una mayor cantidad de cargas y un mayor consumo de energía. 


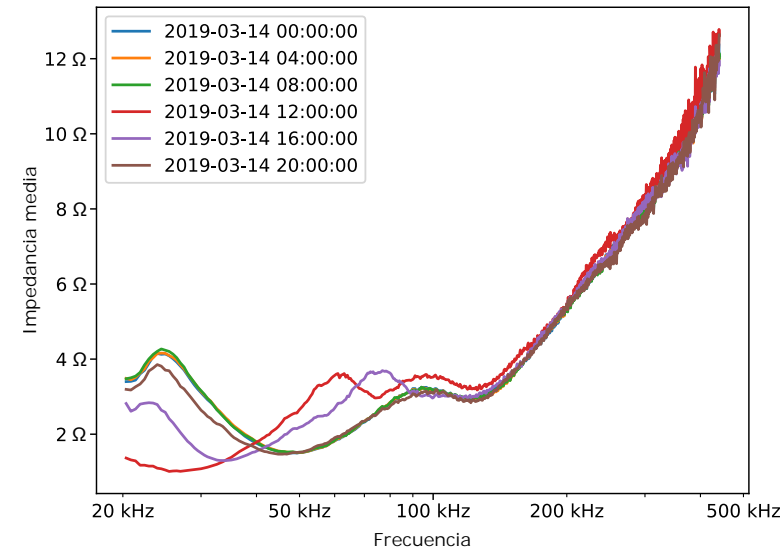

Fig. 7. Módulo de la impedancia media respecto a la frecuencia para un día de semana particular en la primera ubicación.

La Fig. 8 muestra la impedancia mínima respecto a la frecuencia registrada a las 12 pm, para diferentes días. En esta figura se puede observar que el mínimo de impedancia tiene una alta dispersión para la banda superior, teniendo valores mínimos registrados del orden de las decenas de miliohms. Estos valores son prácticamente imposibles de tener en consideración para el diseño, lo que lleva a pérdida de datos cuando se producen estos mínimos, para estas frecuencias. Para frecuencias menores a $85 \mathrm{kHz}$ la impedancia mínima obtenida es del orden de $1 \Omega$.

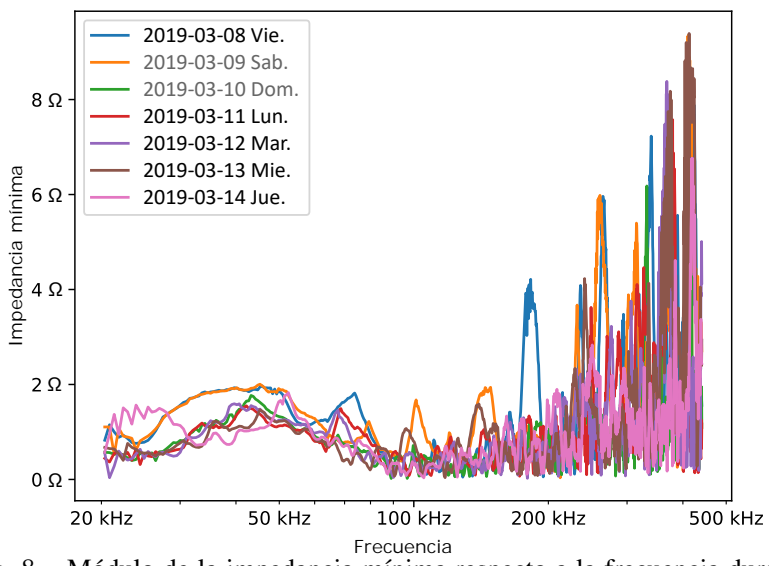

Fig. 8. Módulo de la impedancia mínima respecto a la frecuencia durante una semana medida a las 12 pm para la primera ubicación.

La Fig. 9 muestra la distribución de las impedancias mínimas y medias a lo largo del período de medición. La distribución se divide en frecuencias menores a $150 \mathrm{kHz}$, que corresponden a las bandas CENELEC, y frecuencias mayores a $150 \mathrm{kHz}$, que corresponden a las bandas FCC y ARIB. La tabla I muestra los datos estadísticos de estas distribuciones.
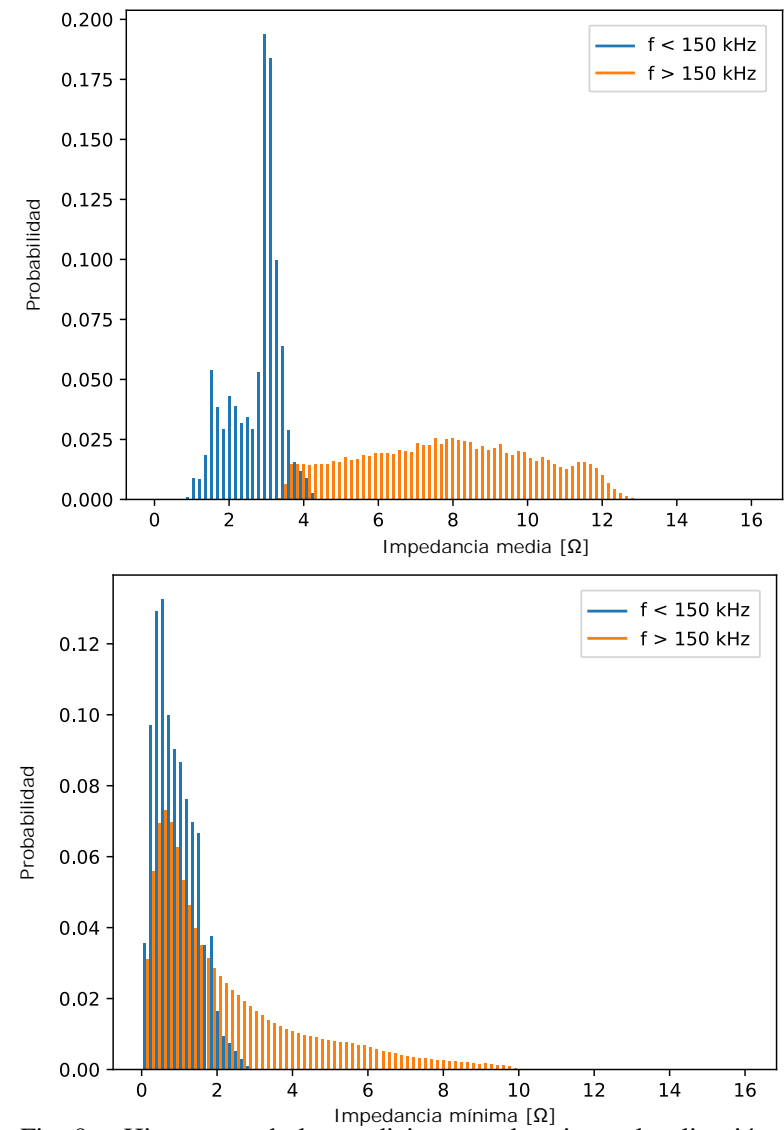

Fig. 9. Histograma de las mediciones en la primera localización.

TABLA I

ESTADÍSTICAS DEL MÓDULO DE LA IMPEDANCIA MEDIDA EN LA PRIMERA UBICACIÓN.

\begin{tabular}{|l|c|c|c|c|}
\hline \multirow{2}{*}{} & \multicolumn{2}{|c|}{$f<150 \mathrm{kHz}$} & \multicolumn{2}{c|}{$f>150 \mathrm{kHz}$} \\
\cline { 2 - 5 } & Media & Mínima & Media & Mínima \\
\hline media & $2.76 \Omega$ & $0.9 \Omega$ & $7.86 \Omega$ & $2.26 \Omega$ \\
\hline mediana & $2.98 \Omega$ & $0.8 \Omega$ & $7.87 \Omega$ & $1.49 \Omega$ \\
\hline moda & $2.99 \Omega$ & $0.53 \Omega$ & $7.9 \Omega$ & $0.6 \Omega$ \\
\hline varianza & 0.45 & $0.3 \Omega$ & 5.34 & $4.19 \Omega$ \\
\hline
\end{tabular}

Del análisis de la Fig. 9 y de la tabla I es evidente que para frecuencias menores a $150 \mathrm{kHz}$ la impedancia predominante es aproximadamente $3 \Omega$, mientras que para frecuencias mayores es aproximadamente $8 \Omega$. También se puede observar que para las bandas CENELEC el valor de la impedancia se encuentra entre $1 \Omega$ y $4 \Omega$, mientras que para las bandas superiores se encuentra entre $3 \Omega$ y $13 \Omega$. Es importante notar que la banda utilizada normalmente para medidores inteligentes es la que presenta menor impedancia media lo que lleva al requerimiento de algoritmos de comunicación más robustos y menores velocidades de transmisión o el sobredimensionamiento del driver del módem.

A pesar que las estadísticas de la impedancia media muestran valores que pueden ser considerados razonables para el diseño, el problema más grave se encuentra en los valores mínimos de impedancia, donde se midieron valores en las decenas de miliohms en ambas bandas, con valores más probables cercanos a los $0.6 \Omega$. La mitad de los valores medidos son menores a $0.8 \Omega$ para las bandas CENELEC y $1.5 \Omega$ para las bandas superiores.

Las Figs. 10 y 11 muestran las impedancias registradas en el segundo emplazamiento de medición, que corresponde 
a un punto de conexión común de todo el laboratorio. Es importante notar que comparando las Figs. 7 y 10 se puede observar que el comportamiento de la impedancia respecto de la frecuencia es completamente diferente. El comportamiento no parece ser completamente inductivo y tiene un máximo local cerca de los $200 \mathrm{kHz}$. Este máximo local es beneficioso para la comunicación, ya que la impedancia media se encuentra en valores similares a los sugeridos por los fabricantes o normativas. Este caso tampoco exhibe un comportamiento como los mostrados por Bausch et.al [14], lo cual puede explicarse por la diferencia entre los ámbitos donde se realizaron los ensayos, como se señaló anteriormente. También se puede notar que esta ubicación no muestra una dependencia temporal, lo que puede ser explicado como un efecto de promediado producto de todas las cargas conectadas en el punto de conexión común. Analizando las impedancias mínimas de la Fig. 11 se observa un comportamiento similar al mostrado en la primera ubicación, con mínimos del orden de las decenas de miliohms.

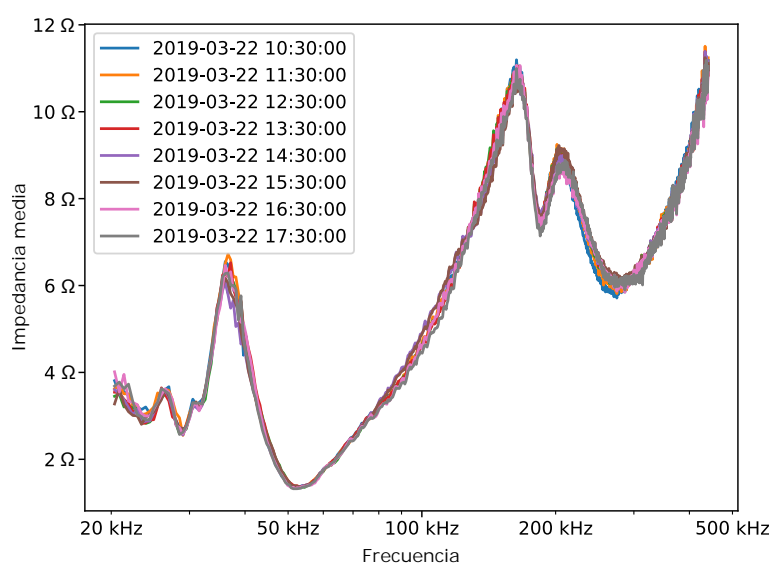

Fig. 10. Módulo de la impedancia media respecto a la frecuencia para diferentes horarios en la segunda ubicación.

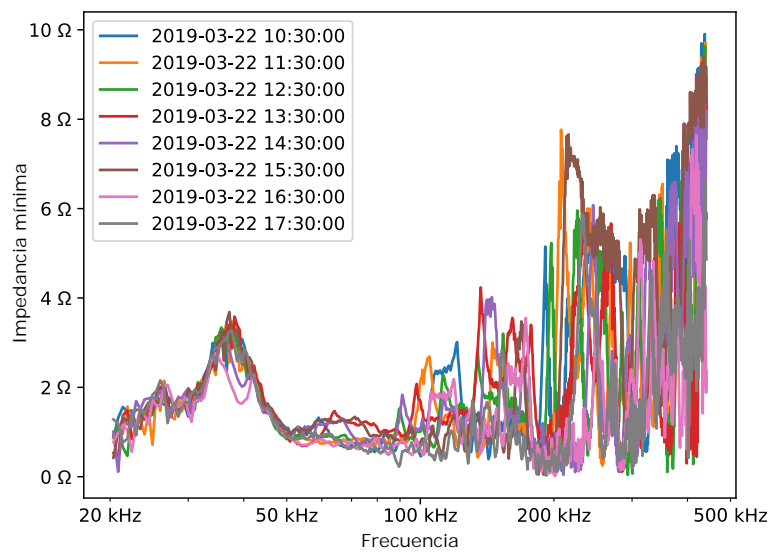

Fig. 11. Módulo de la impedancia mínima respecto a la frecuencia para diferentes horarios en la segunda ubicación.

Finalmente, la Fig. 12 muestra los resultados de la medición en la tercera ubicación. En este caso se puede observar un comportamiento diferente a las otras dos ubicaciones, similar a una red tipo 3 de Bausch et al. [14], que corresponde a tres circuitos resonantes combinados en serie y paralelo. A diferencia de la segunda ubicación, esta medición si presenta dependencia con el tiempo. Es importante remarcar que la impedancia media tiene su mínimo en una frecuencia mayor a las otras dos ubicaciones y este mínimo es cercano a los $0.5 \Omega$. Para este caso, las frecuencias CENELEC muestran valores de impedancia que son bajos para los equipos comerciales pero aceptable desde el punto de vista de diseño, mientras que para frecuencias alrededor de $200 \mathrm{kHz}$ la impedancia cae a niveles que hacen prácticamente imposible una comunicación.

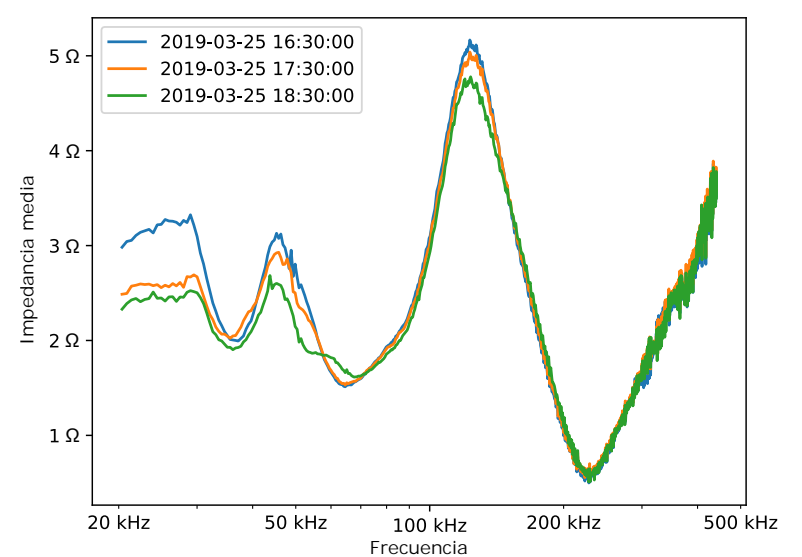

Fig. 12. Módulo de la impedancia media respecto a la frecuencia en diferentes tiempos para la tercera ubicación.

A modo de resumen, la tabla II muestra el rango de las impedancias medias medidas en las tres ubicaciones. Es importante notar que esta tabla muestra valores medios pero las impedancias pueden caer a decenas de miliohms como muestran las Figs. 8 y 11.

TABLA II

RESUMEN DEL RANGO DE LAS IMPEDANCIAS MEDIAS EN LAS TRES UBICACIONES

\begin{tabular}{|l|c|c|}
\hline \multirow{2}{*}{ Ubicación } & \multicolumn{2}{|c|}{ Rango de Impedancia[S] } \\
\cline { 2 - 3 } & $f<150 \mathrm{kHz}$ & Rango Completo \\
\hline Ubicación 1 & $1-5$ & $1-13$ \\
\hline Ubicación 2 & $1-8$ & $1-12$ \\
\hline Ubicación 3 & $1.5-5$ & $0.5-5$ \\
\hline
\end{tabular}

En la Fig. 13 se pueden apreciar las curvas de impedancia media, promediadas a lo largo de todo el período de medición, en los tres emplezamientos de ensayo seleccionados. El comportamiento de la impedancia en todos los casos es diferente, con máyor énfasis en las bandas CENELEC $(<150 \mathrm{kHz})$, que es la banda donde opera la mayoría de los medidores inteligentes. En las frecuencias superiores a $300 \mathrm{kHz}$ se observa un comportamiento similar en todos los casos, predominantemente inductivo, aunque en la tercera ubicación la magnitud de la impedancia es sensiblemente menor a las otras ubicaciones. También es importante destacar la existencia de un máximo local de impedancia en torno a los $200 \mathrm{kHz}$, que solo se manifestó en la segunda ubicación. En ese caso, dado que se trata de la impedancia medida en un tablero principal, al cual se conectan todas las cargas del laboratorio, es posible que ese máximo local se produzca por la interacción de equipos de diferente naturaleza. 


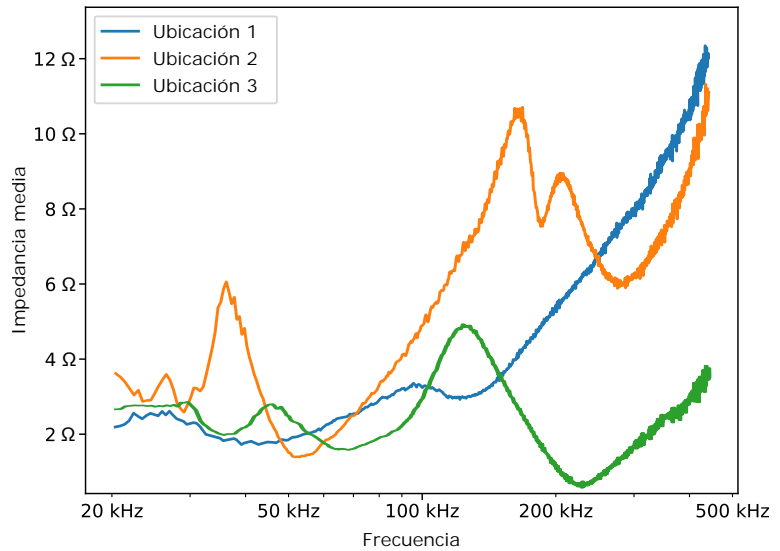

Fig. 13. Promedio del módulo de la impedancia media respecto a la frecuencia para las 3 ubicaciones.

\section{CONCLUSIONES}

En este trabajo se presentaron los resultados de una campaña de medición de la impedancia de PLC de banda angosta en el Laboratorio de Instrumentación y Control del Instituto de Investigaciones Científicas y Tecnológicas en Electrónica (ICYTE). A partir del análisis de los resultados se pudo observar que la impedancia es menor a la prevista por normativas y recomendaciones de diseño de fabricantes, obteniendo impedancias en el rango de las decenas de miliohms. Se pudo verificar también que la impedancia tiene dependencia no solamente del tiempo sino también de la ubicación y cargas conectadas y la magnitud de estas variaciones en ciertas condiciones. Con todo esto, es posible concluir que es importante realizar un análisis de la impedancia para el diseño de los circuitos de comunicación en cada instalación particular, ya que como se describió, una incorrecta estimación de la impedancia puede llevar a problemas de comunicación o costo mayor del necesario. Además, a partir de los resultados se pudo comprobar la hipótesis de que los niveles de impedancia en la red local no son los adecuados para lograr una correcta comunicación utilizando equipamiento comercial, los cuales están normalmente diseñados para operar satisfactoriamente por encima de los $12 \Omega$ dependiendo de la frecuencia utilizada.

\section{AGRADECIMIENTOS}

Este trabajo fue realizado gracias al soporte económico del subsidio de la ANPCyT PICT 2018-02010, al proyecto de unidad ejecutora "Ciudades Inteligentes" PUE 22920180100002CO de CONICET, y la Universidad Nacional de Mar del Plata.

\section{REFERENCIAS}

[1] S. Galli, A. Scaglione, and Z. Wang, "For the grid and through the grid: The role of power line communications in the smart grid," Proceedings of the IEEE, vol. 99, no. 6, pp. 998-1027, June 2011.

[2] "IEEE standard for low-frequency (less than $500 \mathrm{khz}$ ) narrowband power line communications for smart grid applications," IEEE Std 1901.2-2013, pp. 1-269, Dec 2013.

[3] "IEEE standard for low-frequency (less than $500 \mathrm{khz}$ ) narrowband power line communications for smart grid applications - amendment 1," IEEE Std 1901.2a-2015 (Amendment to IEEE Std 1901.2-2013), pp. 1-28, Oct 2015.
[4] N. Andreadou and G. Fulli, "NB-PLC channel: Estimation of periodic impulsive noise parameters and mitigation techniques," International Journal of Electrical Power and Energy Systems, vol. 103, pp. 146 - 158, 2018. [Online]. Available: http://www.sciencedirect.com/science/article/pii/S0142061517332143

[5] M. Nassar, J. Lin, Y. Mortazavi, A. Dabak, I. H. Kim, and B. L. Evans, "Local utility power line communications in the $3-500 \mathrm{kHz}$ band: Channel impairments, noise, and standards," IEEE Signal Processing Magazine, vol. 29, no. 5, pp. 116-127, Sep. 2012.

[6] L. G. da S. Costa, G. R. Colen, A. C. M. de Queiroz, V. L. da Costa, U. R. Vitor, F. V. dos Santos, and M. V. Ribeiro, "Access impedance in brazilian in-home, broadband and low-voltage electric power grids," Electric Power Systems Research, vol. 171, pp. 141 - 149, 2019. [Online]. Available: http://www.sciencedirect.com/science/article/pii/S037877961930080X

[7] H. Mahamudul and C. Ozansoy, "L-C band pass impedance matching coupling circuit for high voltage PLC applications," International Journal of Electrical Power \& Energy Systems, vol. 117, p. 105639, may 2020.

[8] "Signalling on low-voltage electrical installations in the frequency 3 khz to $148,5 \mathrm{khz}$ part 1: General requirements, frequency bands and electromagnetic disturbances," CENELEC EN 50065-1, 2011.

[9] "Instituto de investigaciones científicas y tecnológicas en electrónica," http://icyte.fi.mdp.edu.ar.

[10] P. A. Janse van Rensburg, M. P. Sibanda, and H. C. Ferreira, "Integrated impedance-matching coupler for smart building and other power-line communications applications," IEEE Transactions on Power Delivery, vol. 30, no. 2, pp. 949-956, 2015.

[11] "AFE design for a narrowband power-line communications modem using the AFE031," http://www.ti.com/lit/pdf/sboa130.

[12] I. Cavdar and K. Engin, "Measurements of impedance and attenuation at CENELEC bands for power line communications systems," Sensors, vol. 8, 122008.

[13] J. Cortés, A. Sanz, P. Estopiñán, and J. Garcia, "Analysis of narrowband power line communication channels for advanced metering infrastructure," EURASIP Journal on Advances in Signal Processing, vol. 2015, 032015.

[14] J. Bausch, T. Kistner, M. Babic, and K. Dostert, "Characteristics of indoor power line channels in the frequency range $50-500 \mathrm{khz}$," in 2006 IEEE International Symposium on Power Line Communications and Its Applications, March 2006, pp. 86-91. 